\title{
HYDROGENATED TARGETS FOR HIGH ENERGY PROTON GENERATION FROM LASER IRRADIATION IN THE TNSA REGIME
}

\author{
Lorenzo Torrisi $^{a, *}$, Mariapompea Cutroneo $^{b}$, Jiri Ullschmied $^{c}$ \\ ${ }^{a}$ Physics Department and ES, Messina University, V.le F.S. d'Alcontres 31, 98166 S. Agata, Messina, Italy \\ ${ }^{b}$ Nuclear Physics Institute, ASCR, 25068 Rez, Czech Republic \\ ${ }^{c}$ Institute of Physics, ASCR, v.v.i., 18221 Prague 8, Czech Republic \\ * corresponding author: Itorrisi@unimel.it
}

\begin{abstract}
Polyethylene-based thin targets were irradiated in high vacuum in the TNSA (Target Normal Sheath Acceleration) regime using the PALS laser facility. The plasmais produced in forward direction depending on the laser irradiation conditions, the composition of the target and the geometry. The optical properties of the polymer use nanostructures to increase the laser absorbance. Proton kinetic energies from hundreds $\mathrm{keV}$ up to about $3 \mathrm{MeV}$ were obtained for optimal conditions enhancing the electric field driving the ion acceleration.
\end{abstract}

KEYWORDS: hydrogenated target; proton acceleration; TNSA regime.

\section{INTRODUCTION}

One of the objectives of studies of high-intensity lasergenerating plasmas has been to attain high proton acceleration, and the results are becoming competitive with traditional ion acceleration systems. $\mathrm{MeV}$ proton beams can be applied in various areas of interest, from biomedicine (hadron therapy) to nuclear physics (nuclear fusion processes), from microelectronics (semiconductor doping) to laser ion source (ion injection in accelerators) and to other fields [1, 2. Currently, proton beam acceleration reaches about $50 \mathrm{MeV}$ using $f s$ lasers, but the yield is low and of the order of $10^{11}$ particles/laser shot [3. Due to the low proton yield, the large energy distribution, contamination of the beam with other ions, and high proton emittance, this topic needs further investigation. Using thick hydrogenated targets, such as polymers, Backward Proton Acceleration (BPA), obtainable at laser intensities of the order of $10^{15} \mathrm{~W} / \mathrm{cm}^{2}$, shows the advantages of giving high proton yields and generating a continuum proton current emission using repetitive laser pulses. However BPA has the disadvantage that it accelerates protons at maximum kinetic energies generally below $1 \mathrm{MeV}$ [4. Using Target Normal Sheath Acceleration (TNSA) regime, at intensities of the order of $10^{16}-10^{18} \mathrm{~W} / \mathrm{cm}^{2}$, laser interaction confers higher acceleration and protons may reach energies above $10 \mathrm{MeV}$. However, due to the limited thickness of the target of the order of $1-10 \mu \mathrm{m}$, the proton current remains low especially for the most accelerated ions [5]. Further proton acceleration can be obtained using the Radiation Pressure Acceleration (RPA) regime, at intensities above $10^{18} \mathrm{~W} / \mathrm{cm}^{2}$, at which the ion energies grow significantly but at which protons have high energy spread and low yield emission 6]. Our measurements of proton production by plasma-laser are conducted at laser intensity of the order of $10^{16} \mathrm{~W} / \mathrm{cm}^{2}$ using laser pulses of $300 \mathrm{ps}$ duration, at $1315 \mathrm{~nm}$ wavelength, in order to examine in grater depth the optimization of the composition and the geometry of the target, and even the irradiation conditions for maximizing the proton energy emission using TNSA regime. Measurements are performed using the Asterix laser at the PALS facility, with high pulse reproducibility and rich plasma diagnostics. The targets were based on polyethylene, and a deep investigation was carried out in the order to increase its absorption coefficient with respect to the IR laser wavelength by using nanostructures embedded into the polymer. The laser absorption increment, in fact, permits a great laser energy release to the polymer and to the generated plasma, resulting in higher proton acceleration, as it will be presented and discussed here.

\section{MATERIAL AND METHODS}

The iodine laser at PALS employed for this study operates at a wavelength of $1315 \mathrm{~nm}$, maximum pulse energy of about $600 \mathrm{~J}, 300 \mathrm{ps}$ pulse duration and $70 \mu \mathrm{m}$ spot size. Laser beam was employed as non-polarized and as the "p" or "s" polarized mode. The focal position (FP) of the laser beam with respect to the target surface was fixed to 0 (focal position on the target surface). The targets were based on semicrystalline polyethylene, $\left(\mathrm{CH}_{2}\right)_{n}$, ranging in thickness between $50 \mu \mathrm{m}$ up to $0.5 \mu \mathrm{m}$ with flat surfaces placed in a suitable holder. The targets were prepared diluting polyethylene $(\mathrm{PE})$ powder in xylene at a temperature of $150{ }^{\circ} \mathrm{C}$ in a rotating solution for 1 hour and then depositing the melted mixture on a glass substrate. After solidification, the films were detached from the glass by water floating. In some cases nanoparticles of 


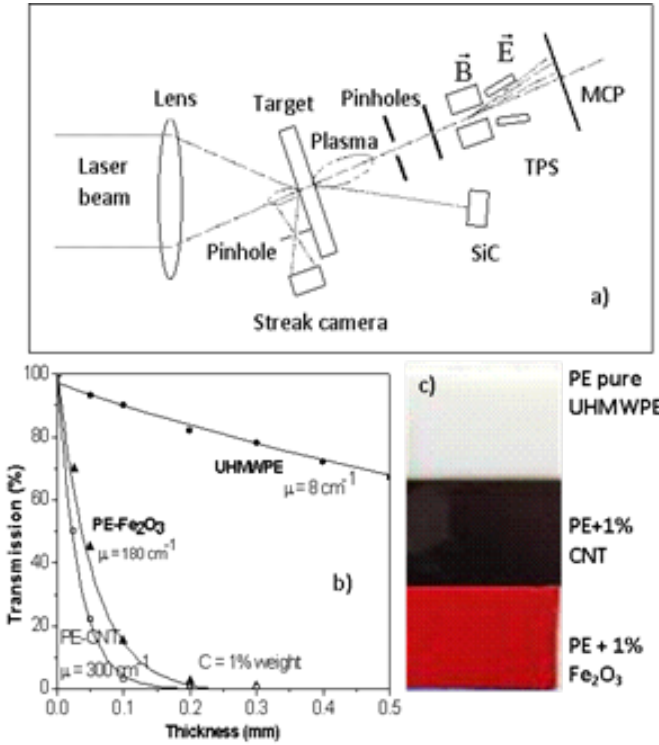

FigURE 1. Experimental set-up (a), transmission vs. thickness at $1315 \mathrm{~nm}$ wavelength of PE pure and containing $1 \% \mathrm{Fe}_{2} \mathrm{O}_{3}$ and CNT nanostructures (b) and a photo of the PE targets that we used (c).

carbon nanotubes (CNT) and $\mathrm{Fe}_{2} \mathrm{O}_{3}$ nanostrucutres at concentrations of $0.1-1 \%$ in weight were introduced into the solution. Pure, thin PE targets appear closely transparent to the visible light, while thick targets and targets containing nanostructures appear opaque and black - colored if the solution contains CNT and red colored if it contains $\mathrm{Fe} 2 \mathrm{O} 3$ (Fig. 1p). The targets were irradiated in the TNSA regime, under $10^{-6}$ mbar vacuum conditions with an incidence angle of $30^{\circ}$. The X-ray CCD streak camera (XSC) was employed to observe the X-ray plasma emission in backward direction. This camera has an exposition time of $2 \mathrm{~ns}$, time resolution of $30 \mathrm{ps}$, spatial resolution of $20 \mu \mathrm{m}$ and spectral range detection efficiency from about $100 \mathrm{eV}$ to $10 \mathrm{keV}$. The calibration correlates the photon energy to false colors from black to red. The ion acceleration was controlled by using a $\mathrm{SiC}$ detector in time-of-flight (TOF) configuration, using a fast, $20 \mathrm{GS} / \mathrm{s}$, storage oscilloscope. The $\mathrm{SiC}$ semiconductor has a gap of $3.2 \mathrm{eV}$, so it is transparent to the visible radiation emitted from hot plasma [7]. It detects UV and X-rays, electrons and ions. Its depletion layer is $80 \mu \mathrm{m}$ in depth at a voltage bias of $600 \mathrm{~V}$. The surface metallization uses $\mathrm{Ni}_{2} \mathrm{Si}$ with $200 \mathrm{~nm}$ thickness, so the minimum detected energy is $30 \mathrm{keV}$ for protons and $150 \mathrm{keV}$ for carbon ions. The $\mathrm{SiC}$ detector was placed in forward direction at an angle of $30^{\circ}$ and a distance of $60 \mathrm{~cm}$ with respect to the normal to the target surface. A Thomson Parabola Spectrometer (TPS) was employed in forward direction along the normal to the target surface at a distance of $133 \mathrm{~cm}$ from the target. Two pin holes, the first of which was $1 \mathrm{~mm}$ in diameter while the second $100 \mu \mathrm{m}$ in diameter, were employed to select only the axial ion component emitted from the plasma.

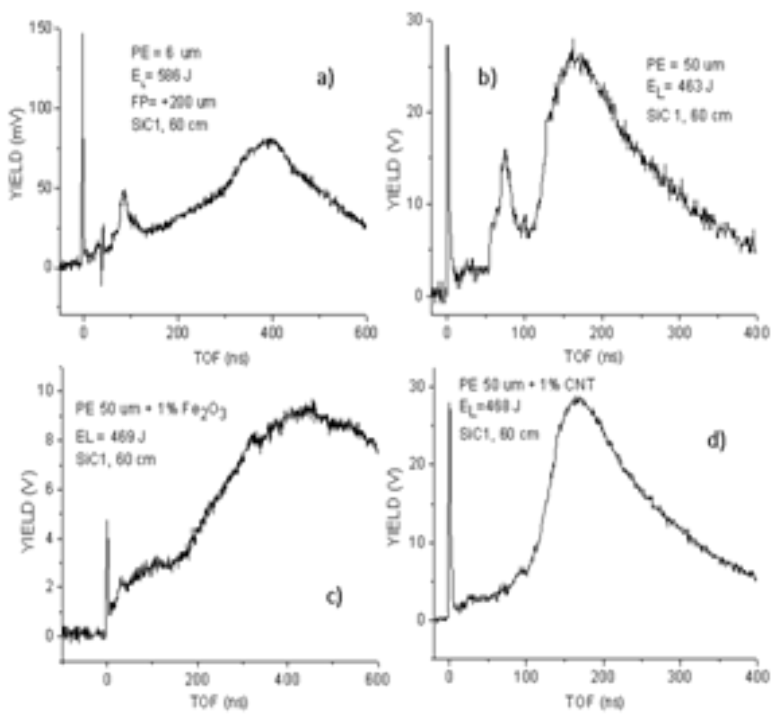

Figure 2. SiC spectra detecting ions from irradiation of $6 \mu \mathrm{m}$ PE (a), $50 \mu \mathrm{m}$ PE pure (b), containing $1 \%$ $\mathrm{Fe}_{2} \mathrm{O}_{3}(\mathrm{c})$ and $\mathrm{CNT}(\mathrm{d})$.

TPS ion deflection uses a $0.06 \mathrm{~T}$ magnetic field and a $1.4 \mathrm{kV} / \mathrm{cm}$ electric field to deflect ions. A multichannel plate (MCP) placed at a distance of $16.5 \mathrm{~cm}$ from the electric field collects the deflected ions, transmits the electrons to the coupled phosphorus screen and enables the images of the parabola to be recorded with a traditional CCD camera. TPS parabolas recognition is performed using Opera 3D Tosca code, in which ion trajectories of various charge-to-mass ratios and energies are simulated and projected, using the real geometry and values of the deflecting fields, on the orthogonal MCP plane, as presented in previous paper [8. Fig. 17 shows a scheme of the experimental set-up.

\section{Results And Discussion}

Fig. 2 a reports a typical SiC-TOF spectrum obtained by irradiating a pure PE thin film, $6 \mu \mathrm{m}$ in thickness, with $586 \mathrm{~J}$ pulse energy. The spectrum shows a photopeak due to the detection of UV and X-rays, representing the start of the TOF analysis, the background signal due to the electrons and their Bremsstrahlung and a larger peak due to protons and carbon ions of the plasma that is produced. The faster protons, which occur during the rapid ascent of the peak at $77 \mathrm{~ns}$, correspond to a kinetic energy of $300 \mathrm{keV}$ and the subsequent faster carbon ions, which occur at about $135 \mathrm{~ns}$, correspond to maximum kinetic energy of about $1.23 \mathrm{MeV}$. This low ion acceleration in TNSA regime is justified by the low laser energy deposition in the pure polymer. $\mathrm{PE}$, in fact, has very low absorption at the laser IR wavelength, according to the transmission measurements reported in the plot of Fig. 1p, indicating high transmission, of about $90 \%$, at $1.3 \mu \mathrm{m}$ wavelength for a thickness of $100 \mu \mathrm{m}$. At 


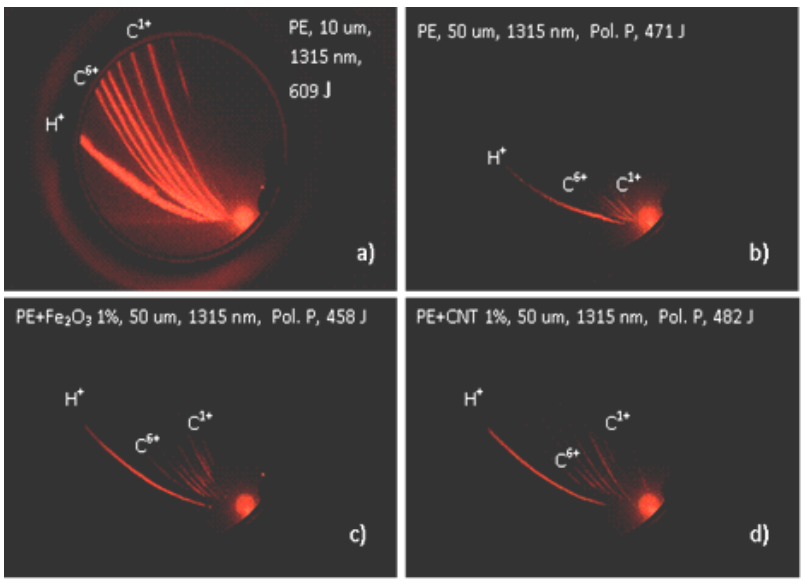

FIGURE 3. TPS spectra relative to ion detection from $10 \mu \mathrm{m} \mathrm{PE} \mathrm{(a)} \mathrm{and} 50 \mu \mathrm{m} \mathrm{PE}$ as pure (b) and containing $1 \%$ of $\mathrm{Fe}_{2} \mathrm{O}_{3}$ (c) and CNT (d).

this wavelength the absorption coefficient, calculable using the Lambert-Beer law, is $8 \mathrm{~cm}^{-1}$ [9]. Using a target of $6 \mu \mathrm{m}$ in thickness the transmitted radiation is therefore about $99 \%$ and the absorbed radiation only $1 \%$.

The SiC-TOF spectrum of Fig. $2 \mathrm{p}$ shows the accelerated ions irradiating a $5 \mu \mathrm{m} \mathrm{PE}$ target with a $463 \mathrm{~J}$ laser pulse. In this case, although the laser pulse energy is about $21 \%$ lower, faster protons and carbon ions are produced, occurring at a TOF of $69 \mathrm{~ns}$ and $120 \mathrm{~ns}$, corresponding to kinetic energy of $400 \mathrm{keV}$ and $1.6 \mathrm{MeV}$, respectively. This result is increased to about $5 \%$, due to the increment of the absorbed laser energy in the target, and consequently due to the hotter plasma that is generated. A further increment of the laser absorption in PE can be obtained using highly absorbent nanostructures embedded in the polymer. This is the case for $\mathrm{Fe}_{2} \mathrm{O}_{3}$ and CNT embedded uniformly in the polymer at concentrations of the order of $1 \%$ in weight, which significantly changes the chemical and physical properties of the polymer. Fig. 22 shows a typical SiC-TOF spectrum of PE, $50 \mu \mathrm{m}$ in thickness, containing $\mathrm{Fe}_{2} \mathrm{O}_{3}$ nanostructures, irradiated at $469 \mathrm{~J}$. In this case, the spectrum differs significantly from the spectrum for pure PE, showing ion peaks occurring at lower TOF. Protons have a minimum TOF of about $33 \mathrm{~ns}$, corresponding to a maximum kinetic energy of $1.72 \mathrm{MeV}$, while $\mathrm{C}$ ions have a maximum energy of $3.5 \mathrm{MeV}$. This result can be justified on the basis of the high absorption coefficient of the polymer which, with the $\mathrm{Fe}_{2} \mathrm{O}_{3}$ nanostructures, permits an absorption level of about $53 \%$. Using a $\mathrm{PE}$ thin film $50 \mu \mathrm{m}$ in thickness, containing CNT at $1 \%$ concentration, the foil absorbance increases up to $78 \%$. Higher ion acceleration is therefore expected, as reported in Fig. $2 \mathrm{~d}$ for PE irradiated at $468 \mathrm{~J}$. In this case, the protons have a minimum TOF of about $25 \mathrm{~ns}$ corresponding to a maximum kinetic energy of $2.99 \mathrm{MeV}$ and the carbon ions have maximum energy of $5.5 \mathrm{MeV}$. TPS spectra provide further information
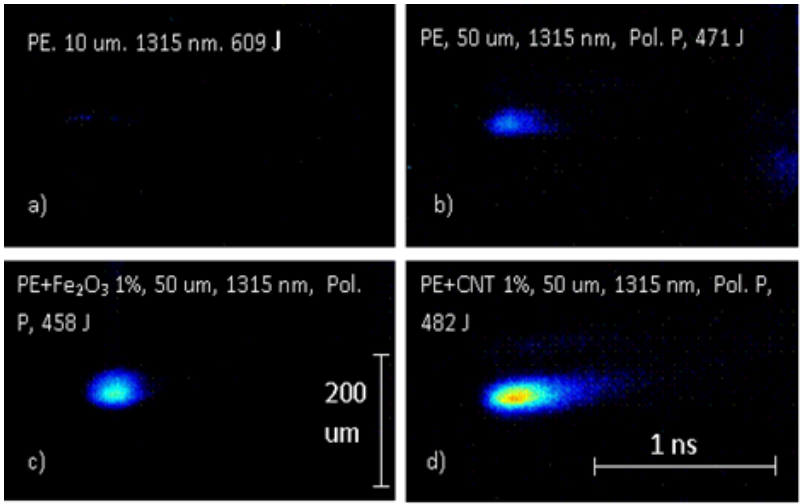

FiguRE 4. X-ray streak camera images relative to plasma detection from $10 \mu \mathrm{m} \mathrm{PE} \mathrm{(a),} \mathrm{from} 50 \mu \mathrm{m}$ PE as pure (b), containing $1 \%$ of $\mathrm{Fe}_{2} \mathrm{O}_{3}$ (c) and containing $1 \%$ of $\mathrm{CNT}(\mathrm{d})$.

on the plasma produced in forward direction. Fig. 3 shows typical TPS images obtained detecting the ion emission from a PE foil $10 \mu \mathrm{m}$ in thickness (a) and $50 \mu \mathrm{m}$ in thickness (b). For comparison, the spectra obtained when irradiating $50 \mu \mathrm{m}$ PE with embedded $\mathrm{Fe}_{2} \mathrm{O}_{3}$ (c) and with embedded CNT (d) are also reported. The obtained parabolas are in relation to the protons and carbon ions coming from the polymer composition. For thick PE foils, the luminosity of the parabola decreases due to the lower transmitted ions, and only the most energetic ions, which are less deflected, are detected. Using the embedded nanostructures, the ions increase their energy and their parabolas appear nearer to the non-deflection center.

However the weak luminosity indicates that there is only a small number of more accelerated ions, and in some cases this means that the maximum ion energy for protons and carbon ions cannot be evaluated accurately. From this point of view, the $\mathrm{SiC}$ detector seems to be more sensitive to the ion energetic measurement with respect to TPS. Further details on the plasma that is produced can be obtained by analyzing the $\mathrm{X}$-ray streak camera images. As an example Fig. 4 shows the XSC images obtained by irradiating a PE foil $1 \mu \mathrm{m}$ in thickness (a) and $50 \mu \mathrm{m}$ in thickness (b).

In the first case, cold plasma is obtained, because the laser absorption in the foil is negligible and no $\mathrm{X}$-rays are emitted, while in the second case the laser absorption increases and plasma emitting low energetic X-rays is produced. For comparison the Fig. 44. and Fig. $4 d$ present the plasma obtained by irradiating $\mathrm{PE}$ in which $1 \% \mathrm{Fe}_{2} \mathrm{O}_{3}$ is embedded and $\mathrm{PE}$ in which $1 \% \mathrm{CNT}$ is embedded respectively. The higher XSC light intensity (false colors proportional to the plasma temperature) demonstrates that in this last case the plasma emits more energetic X-ray as result of the higher laser absorption in the thin nanostructured target. 


\section{Conclusions}

Hydrogenated targets can be used for high energy proton acceleration from laser irradiation in the TNSA regime. Polyethylene can be employed to generate high energetic and high yield protons only if its thickness is in the range $6-50 \mu \mathrm{m}$ and if it contains absorbent nanostructures such as $\mathrm{Fe}_{2} \mathrm{O}_{3}$ and CNT at concentrations of about $1 \%$. This last mentioned structure has the advantage that it absorbs high hydrogen content and to develops high proton emission as a consequence of the laser-generated plasma.

\section{ACKNOWLEDGEMENTS}

The research leading to these results has received funding from LASERLAB-EUROPE (Grant agreement 284464, EC's Seventh Framework Programme)

\section{REFERENCES}

[1] G. A. P. Cirrone, M. Carpinelli, G. Cuttone, al. Elimed, future hadrontherapy applications of laser-accelerated beams. Nucl Instr and Methods A 730:174-177, 2013.

[2] L. Torrisi, S. Cavallaro, M. Cutroneo, al. Deuteriumdeuterium nuclear reaction induced by high intensity laser pulses. L Torrisi, S Cavallaro, M Cutroneo et Al, Applied Surface Sci 272 (2013) 42-45 272:42-45, 2013.
[3] A. Macchi, M. Borghesi, M. Passoni. Ion acceleration by super-intense laser-plasma interaction. Plasma Physics 1775:1-58, 2013.

[4] L. Torrisi, G. Foti, L. Giuffrida, al. Single crystal silicon carbide detector of emitted ions and soft $\mathrm{x}$ rays from power laser-generated plasmas. J Appl Phys 105:123304, 2009.

[5] J. Badziak, S. Glowacz, S. Jablonski, al. Production of ultrahigh ion current densities at skin-layer subrelativistic laser-plasma interaction. Plasma Phys Contr Fus 46(12B):044, 2004.

[6] A. P. L. Robinson, M. Zepf, S. Kar, al. Radiation pressure acceleration of thin foils with circularly polarized laser pulses. New Journal of Physics 10:1367, 2008.

[7] M. Cutroneo, P. Musumeci, M. Zimbone, al. High performance sic detectors for mev ion beamsgenerated by intense pulsed laser plasmas. $M$ Cutroneo, $P$ Musumeci, $M$ Zimbone et Al, J Material Research 28(01), pp 87 â̆ $\breve{S}$ 93, 2012 28(01):87-93, 2012.

[8] M. Cutroneo, L. Torrisi, L. Andó, al. Thomson parabola spectrometerfor energetic ions emitted from sub-ns laser generated plasmas. Acta Polytechnica 53(2):138-141, 2013.

[9] L. Torrisi, F. Caridi, A. M. Visco, al. Polyethylene welding by pulsed visible laser irradiation. Appl Surf Science 257:2567âĂŞ2575, 2011. 